\title{
An innovative method to calibrate a spinner anemometer without the use of yaw position sensor
}

\author{
Giorgio Demurtas ${ }^{1}$ and Nick Gerardus Cornelis Janssen ${ }^{2}$ \\ ${ }^{1}$ DTU Wind Energy, Frederiksborvej 399, 4000 Roskilde, Denmark \\ ${ }^{2}$ Romo Wind A/S, Olof Palmes Alle 47, 8200 Aarhus N, Denmark
}

Correspondence to: Giorgio Demurtas (giod@dtu.dk)

Received: 12 April 2016 - Published in Wind Energ. Sci. Discuss.: 19 April 2016

Revised: 18 July 2016 - Accepted: 7 September 2016 - Published: 27 September 2016

\begin{abstract}
A spinner anemometer can be used to measure the yaw misalignment and flow inclination experienced by a wind turbine. Previous calibration methods used to calibrate a spinner anemometer for flow angle measurements were based on measurements of a spinner anemometer with default settings (arbitrary values, generally $k_{1, \mathrm{~d}}=1$ and $k_{2, \mathrm{~d}}=1$ ) and a reference yaw misalignment signal measured with a yaw position sensor. The yaw position sensor is normally present in wind turbines for control purposes; however, such a signal is not always available for a spinner anemometer calibration. Therefore, an additional yaw position sensor was installed prior to the spinner anemometer calibration. An innovative method to calibrate the spinner anemometer without a yaw positions sensor was then developed. It was noted that a non-calibrated spinner anemometer that overestimates (underestimates) the inflow angle will also overestimate (underestimate) the wind speed when there is a yaw misalignment. The new method leverages the non-linearity of the spinner anemometer algorithm to find the calibration factor $F_{\alpha}$ by an optimization process that minimizes the dependency of the wind speed on the yaw misalignment. The new calibration method was found to be rather robust, with $F_{\alpha}$ values within $\pm 2.7 \%$ of the mean value for four successive tests at the same rotor position.
\end{abstract}

\section{Introduction}

The spinner anemometer (Pedersen et al., 2007) measures the horizontal wind speed $U_{\text {hor }}$, yaw misalignment $\gamma$ and flow inclination $\beta$ experienced by a wind turbine by measuring the flow on the spinner by using three 1-D sonic sensors. The three 1-D sonic sensors are mounted on the spinner and connected to a so-called "conversion box". Each sonic sensor arm also contains a 1-D accelerometer, the measurements of which are used in the conversion box to calculate the rotor position. The main purpose of the conversion box is to execute the conversion algorithm that transforms the 1-D sonic sensor readings, which are in a rotating coordinate reference system (Fig. 1), to the fixed nacelle coordinate reference system as $U_{\text {hor }}, \gamma$ and $\beta$. The conversion algorithm takes into consideration the wind turbine tilt angle $\delta$, which is set in the conversion box as a constant. The shape of the spinner is accounted for by two calibration coefficients: $k_{1}$ and $k_{2}$. The first coefficient mainly relates to wind speed measurements, while the ratio of the two coefficients $k_{\alpha}=k_{2} / k_{1}$ mainly relates to flow angle measurements. The relations between the wind speed $U$, flow angle $\alpha$ and azimuth position of the stagnation point $\theta$ producing $V_{1}, V_{2}$ and $V_{3}$ measured by the three 1-D sonic sensors are

$$
\begin{aligned}
V_{1} & =U\left(k_{1} \cos (\alpha)-k_{2} \sin (\alpha) \cos (\theta)\right) \\
& =U \cdot k_{1}\left(\cos (\alpha)-k_{\alpha} \sin (\alpha) \cos (\theta)\right), \\
V_{2} & =U\left(k_{1} \cos (\alpha)-k_{2} \sin (\alpha) \cos \left(\theta-\frac{2 \pi}{3}\right)\right) \\
& =U \cdot k_{1}\left(\cos (\alpha)-k_{\alpha} \sin (\alpha) \cos \left(\theta-\frac{2 \pi}{3}\right)\right),
\end{aligned}
$$




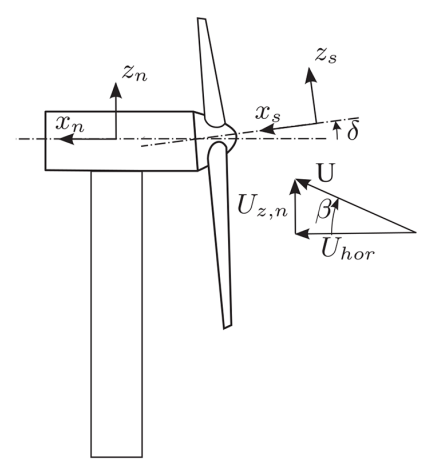

(a) Side view

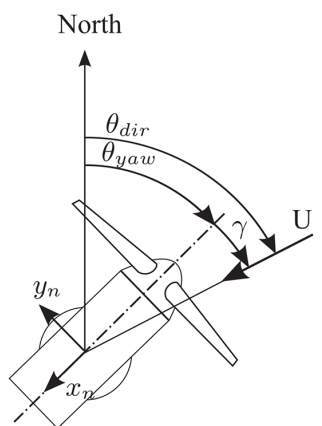

(b) Top view

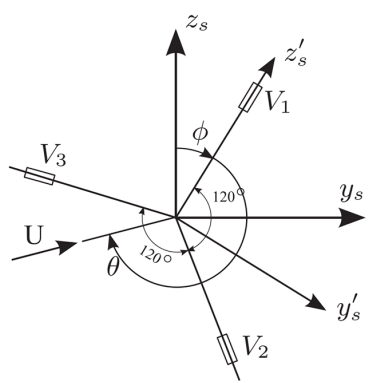

(c) Front view along the shaft axis

Figure 1. Coordinate systems and definition of angles: rotating spinner coordinate system $x_{\mathrm{s}}^{\prime}, y_{\mathrm{s}}^{\prime}$ and $z_{\mathrm{s}}^{\prime}$; non-rotating shaft coordinate system $x_{\mathrm{s}}, y_{\mathrm{s}}$ and $z_{\mathrm{s}}$; fixed nacelle coordinate system $x_{\mathrm{n}}, y_{\mathrm{n}}$ and $z_{\mathrm{n}}$; yaw direction $\theta_{\text {yaw }}$; yaw misalignment $\gamma$; flow inclination angle $\beta$; tilt angle $\delta$; azimuth position of flow stagnation point on spinner $\theta$ (relative to sonic sensor 1) and rotor azimuth position $\phi$ (position of sonic sensor 1 relative to vertical). From Demurtas et al. (2016).

$$
\begin{aligned}
V_{3} & =U\left(k_{1} \cos (\alpha)-k_{2} \sin (\alpha) \cos \left(\theta-\frac{4 \pi}{3}\right)\right) \\
& =U \cdot k_{1}\left(\cos (\alpha)-k_{\alpha} \sin (\alpha) \cos \left(\theta-\frac{4 \pi}{3}\right)\right)
\end{aligned}
$$

The conversion algorithm (Eqs. 5 to 18 ) was derived from Eqs. (1) to (3). The values of $k_{1}$ and $k_{2}$ constants are generally not know when the spinner anemometer is installed on a wind turbine for the first time; they are therefore set to an arbitrary value, generally $k_{1, \mathrm{~d}}=1$ and $k_{2, \mathrm{~d}}=1$. The calibration procedure will then provide the correction factors $F_{1}$ and $F_{\alpha}$ to correct the default values to calibrated values (Eq. 4). The output values relative to a spinner anemometer which measures with default calibration settings has the subscript " $d$ " $\left(U_{\text {hor,d }}, \gamma_{\mathrm{d}}, \beta_{\mathrm{d}}\right)$.

$$
\begin{aligned}
& k_{1}=F_{1} \cdot k_{1, \mathrm{~d}} \quad k_{2}=F_{2} \cdot k_{2, \mathrm{~d}}=k_{\alpha} \cdot k_{1}=k_{\alpha, \mathrm{d}} \cdot F_{\alpha} \cdot k_{1} \\
& \alpha=\arctan \left(\frac{k_{1} \sqrt{3\left(V_{1}-V_{\mathrm{ave}}\right)^{2}+\left(V_{2}-V_{3}\right)^{2}}}{\sqrt{3} k_{2} V_{\mathrm{ave}}}\right) \\
& V_{\mathrm{ave}}=\frac{1}{3}\left(V_{1}+V_{2}+V_{3}\right) \\
& U=\frac{V_{\mathrm{ave}}}{k_{1} \cos \alpha} \\
& V_{1}<V_{\mathrm{ave}}: \theta=\arctan \frac{\left(V_{2}-V_{3}\right)}{\sqrt{3}\left(V_{1}-V_{\mathrm{ave}}\right)} \\
& V_{1} \geq V_{\mathrm{ave}}: \theta=\arctan \frac{\left(V_{2}-V_{3}\right)}{\sqrt{3}\left(V_{1}-V_{\mathrm{ave}}\right)}+\pi \\
& U_{x, \mathrm{~s}}=U \cos (\alpha) \\
& U_{\alpha}=U \sin (\alpha) \\
& U_{y, \mathrm{~s}}=-U_{\alpha} \sin (\phi+\theta) \\
& U_{z, \mathrm{~s}}=-U_{\alpha} \cos (\phi+\theta) \\
& U_{x}=U_{x, \mathrm{~s}} \cos (\delta)+U_{z, \mathrm{~s}} \sin (\delta)
\end{aligned}
$$

$$
\begin{aligned}
& U_{y}=U_{y, \mathrm{~s}} \\
& U_{z}=U_{z, \mathrm{~s}} \cos (\delta)-U_{x, \mathrm{~s}} \sin (\delta) \\
& U_{\text {hor }}=\sqrt{U_{x}^{2}+U_{y}^{2}} \\
& \gamma=\arctan \left(\frac{U_{y}}{U_{x}}\right) \\
& \beta=\arctan \left(\frac{U_{z}}{U_{\mathrm{hor}}}\right)
\end{aligned}
$$

\section{Existing calibration methods for flow angle measurements}

Two methods based on measurements to calibrate a spinner anemometer for flow angle measurements proposed in Pedersen and Demurtas (2014) consist of yawing the wind turbine by $\pm 60^{\circ}$ several times under manual control (as indicated by the turbine yaw position sensor, with respect to the mean wind direction). During this test, the output parameters of the spinner anemometer $\left(U_{\text {hor }}, \gamma, \beta\right)$ are recorded at a high sampling frequency $(10 \mathrm{~Hz})$. The analysis of the measurements provides the correction factor $F_{\alpha}$ that, multiplied by the default $k_{\alpha, \mathrm{d}}$, gives the correct $k_{\alpha}$ calibration value.

The methods are based on the assumption that the wind direction is constant during the test. Due to this requirement, Pedersen and Demurtas (2014) recommended doing the test at wind speeds above $6 \mathrm{~m} \mathrm{~s}^{-1}$. Both methods need the yaw position to be measured in order to calculate the reference yaw misalignment $\gamma_{\text {ref }}$, defined as the mean wind direction minus the instantaneous yaw position during the test (see Pedersen and Demurtas (2014) for details). In the first method (abbreviated as GGref), $F_{\alpha}$ was calculated by calibrating the measurements iteratively, until the linear fit of $\gamma$ as a function of $\gamma_{\text {ref }}$ was giving a line of slope equal to 1 .

In the second method (abbreviated as TanTan), only one linear fitting was made to $\tan (\gamma)$ as a function of $\tan \left(\gamma_{\mathrm{ref}}\right)$. In this case, the slope coefficient of the fit was exactly $F_{\alpha}$. The 
two calibration methods were found to be sensitive to the width of the yawing span. In fact, different $F_{\alpha}$ values were obtained, subsetting the data set to a variable span of $\gamma_{\text {ref. }}$.

A new method to find the $F_{\alpha}$ value that does not require a yaw position measurement and to use the non-linearity of the spinner anemometer conversion algorithm is proposed.

\section{The wind speed response method}

The wind speed response method (abbreviated WSR) is based on the assumption that the wind speed is constant during the test. The turbulence of the real wind will add some scatter in the measurements which will reduce the repeatability of the result. While in principle a single yawing movement is sufficient, in practice the wind speed fluctuations need to be averaged by yawing the wind turbine several times. The spinner anemometer is able to measure inflow angles (yaw misalignment $\gamma$ and flow inclination $\beta$ ) and wind speed $U$. A wrong $k_{\alpha}$ value will result in a wrong value of the angle $\gamma$, which will turn into a wrong value of the horizontal wind speed $U_{\text {hor }}$. In other words, a wrong $k_{\alpha}$ makes the wind speed measurement dependent on the yaw misalignment. This property of the spinner anemometer model (Eqs. 1-3) was verified with a data set consisting of constant wind speed $U_{\text {hor }}$ and 13 values of yaw misalignment going from -60 to $60^{\circ}$ in steps of $10^{\circ}$. The tilt angle and the flow inclination were set to arbitrary values (equal to zero for Fig. 2). In the real world the tilt angle of the wind turbine is typically between 3 and $6^{\circ}$, while the flow inclination varies within approximately $\pm 10^{\circ}$. The conversion algorithm takes into consideration both the tilt angle $\delta$ and the measured flow inclination $\beta_{\mathrm{d}}$ when calculating the yaw misalignment $\gamma_{\mathrm{d}}$; therefore, they have no influence on the result of this method. $V_{1}, V_{2}$ and $V_{3}$ were calculated with Eqs. (1)-(3) with $k_{\alpha}=1$ and $k_{2}=1$.

Equations (5) to (18) (which are the direct conversion algorithm presented in Pedersen and Demurtas, 2014) were used with new values of $k_{\alpha}$ equal to $0.5,1$ and 2, with the calculated $V_{1}, V_{2}$ and $V_{3}$ to calculate $U_{\text {hor,d }}$ and $\alpha_{\mathrm{d}} . k_{1}$ was kept equal to 1 .

When the conversion was made with $k_{\alpha}=1, U_{\text {hor,d }}$ and $\alpha_{\mathrm{d}}$ matched the (correct) initial values of $U_{\text {hor }}$ and $\alpha$ (black line in Fig. 2). On the other hand, when the conversion was made with $k_{\alpha, \mathrm{d}}=0.5$, the wind speed and angle were overestimated (blue curve in Fig. 2) because $k_{\alpha, \mathrm{d}}$ is too small compared to the correct $k_{\alpha}$ value equal to 1 in this example. Similarly, with $k_{\alpha, \mathrm{d}}=2$, the angles and the wind speed were underestimated (red curve in Fig. 2).

From the experience of calibration on several turbines, the default settings of $k_{\alpha, \mathrm{d}}=1$ is too small. Therefore, the wind speed response looks like a happy smile, and an $F_{\alpha}>1$ is required to correct the default calibration value. Note that the wind speed is still measured correctly for a small inflow angle (where the three curves of Fig. 2 are close to each other).

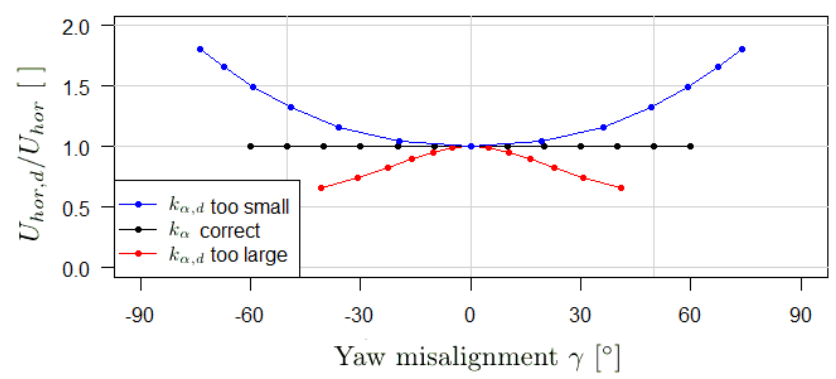

Figure 2. Effect of three $k_{\alpha}$ values on yaw misalignment and wind speed measurements. Black line shows data where the $k_{\alpha}$ is correct (equal to 1 for our theoretical spinner model). Blue curve shows $k_{\alpha}$ set to 0.5 . To correct the blue curve to the black curve, the correction should be made with $F_{\alpha}>1$ ( $F_{\alpha}=2$ in this case). Red line shows $k_{\alpha}$ set to twice the correct value; therefore, we need $F_{\alpha}<1$ to correct the measurements to the black line.

The method to optimize $F_{\alpha}$ consists of minimizing the RMSE (root mean square error) of a horizontal linear fit made to the measurements of $U_{\text {hor,d }}$ for varying $F_{\alpha}$. $U_{\text {hor }}$ is obtained applying the $F_{\alpha}$ calibration to the measurements of $U_{\text {hor,d }}, \gamma_{\mathrm{d}}$ and $\beta_{\mathrm{d}}$ acquired with default values $k_{1, \mathrm{~d}}$ and $k_{2, \mathrm{~d}}$. For this reason $U_{\text {hor }}$ is a function of $U_{\text {hor,d }}, \gamma_{\mathrm{d}}, \beta_{\mathrm{d}}, k_{1, \mathrm{~d}}, k_{2, \mathrm{~d}}$ and $F_{\alpha}$.

The function object of the optimization is

$$
\begin{aligned}
\mathrm{RMSE} & =f\left(U_{\mathrm{hor}, \mathrm{d}}, \gamma_{\mathrm{d}}, \beta_{\mathrm{d}}, k_{1, \mathrm{~d}}, k_{2, \mathrm{~d}}, F_{\alpha}\right) \\
& =\sqrt{\frac{1}{n} \sum_{1}^{n}\left(\overline{U_{\mathrm{hor}}}-U_{\mathrm{hor}}\right)^{2}},
\end{aligned}
$$

where the first three variables come from the measurements, the fourth and fifth are the settings of the spinner anemometer at the time of acquisition of the measurements, and the last one $\left(F_{\alpha}\right)$ is the independent variable used in the optimization. The function of Eq. (19) was optimized to its minimum using a combination of golden section search and successive parabolic interpolation (Brent, 1973).

\section{Application of the method}

The measurements were acquired in February 2016 on a NEG Micon $2 \mathrm{MW}$ wind turbine installed in Denmark. The wind turbine was yawed in and out of the wind several times with the rotor stopped with one blade pointing downwards. Figures 3 and 4 show the $10 \mathrm{~Hz}$ data recorded during the calibration procedure. Figure $3 \mathrm{a}-\mathrm{c}$ show non-calibrated measurements, while Fig. $4 \mathrm{a}-\mathrm{c}$ show calibrated measurements. In both Figs. 3 and 4, the sub-figure (a) shows the time series of the yaw misalignment and yaw misalignment reference (measured with a yaw position sensor). Sub-figure (b) shows the time series of the wind speed. Sub-figure (c) shows the wind speed response as a function of yaw misalignment. 

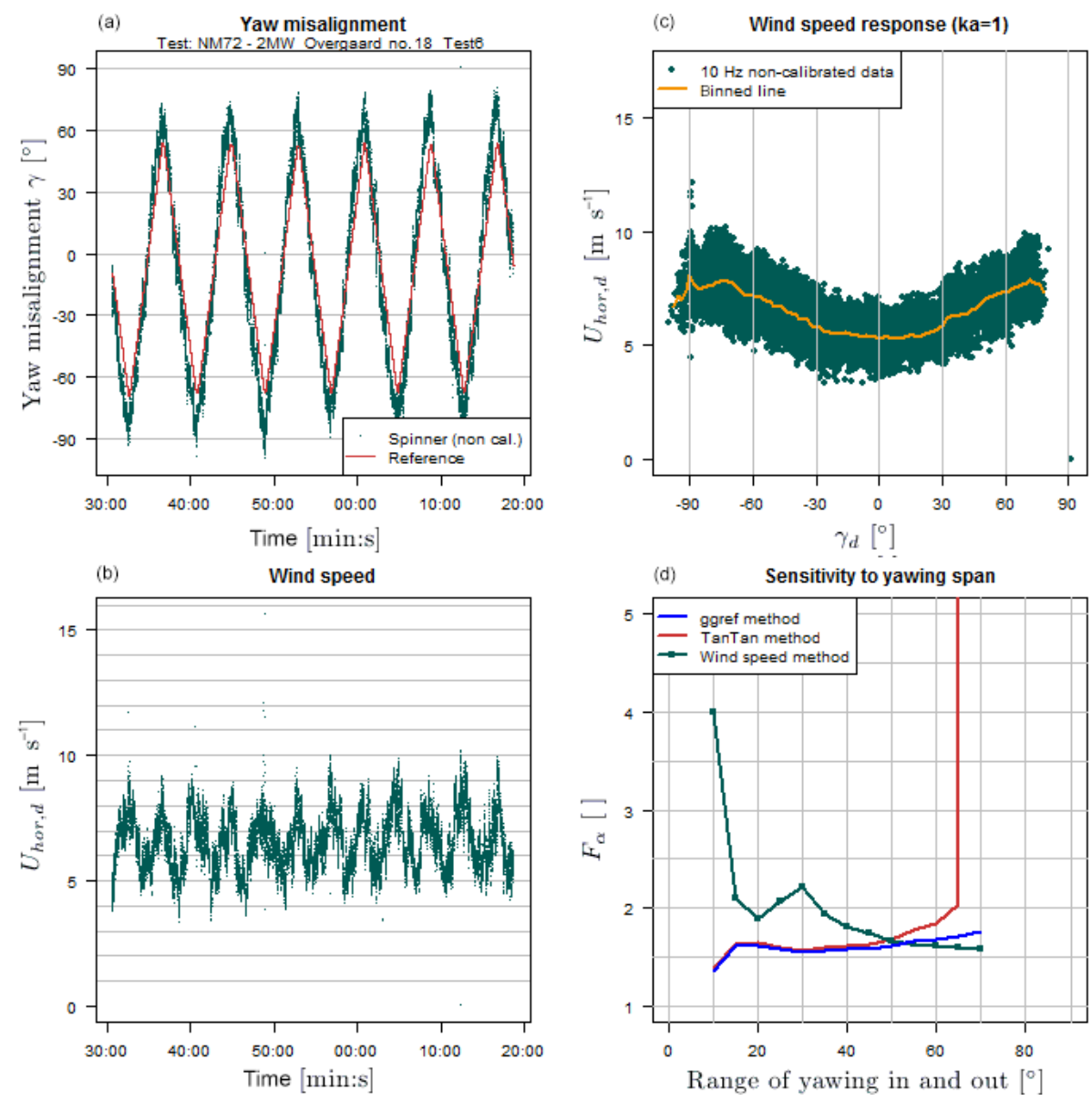

Figure 3. Before calibration, test 6. (a) Time series of yaw misalignment as measured by the spinner anemometer and by the yaw position sensor. (b) Wind speed time series as measured by the spinner anemometer before $F_{1}$ calibration. (c) Wind speed as a function of yaw misalignment measured by spinner anemometer. (d) Calibration correction factor $F_{\alpha}$ calculated in three different methods as a function of yawing span ranging from \pm 10 to $\pm 90^{\circ}$ in steps of $\pm 5^{\circ}$.

Figure 3d shows the value of $F_{\alpha}$ calculated with the three different methods (GGref and TanTan from Pedersen and Demurtas (2014) and the present method, WSR), for varying ranges of yawing the wind turbine out of the wind (data were filtered according to $\gamma_{\text {ref }}$ in steps of $5^{\circ}$ span per side). The $F_{\alpha}$ value was calculated with the WSR method only if there were at least $30 \mathrm{~s}$ of measurements in the outmost $5^{\circ}$ of the considered range (which justifies the fact that the scatter plot of Fig. $4 \mathrm{c}$ appears wider than the maximum range shown in Fig. $3 \mathrm{~d}$ by the green line).

\section{Discussion}

As seen also in tests performed on other wind turbine models, the GGref and TanTan methods tend to give a higher $F_{\alpha}$ for increasing yawing span than the WSR method. This is especially true for the TanTan method because of the tangent function properties, which tend to increase rapidly when approaching a $90^{\circ}$ angle.
As seen in Fig. 3d, the value of $F_{\alpha}$ is dependent on the chosen width of yawing the turbine in and out of the wind. For the TanTan and GGref methods, Pedersen and Demurtas (2014) suggested limiting the span to $\pm 45^{\circ}$. The value of $F_{\alpha}$ calculated with the WSR method tends to stabilize and be comparable with the previous two methods for a yawing span within 50 and $70^{\circ}$.

Above a certain large inflow angle (depending on the spinner shape) the air flow would separate from the spinner surface with the consequence of the downwind sensor measuring in a separated flow region. In this condition the spinner anemometer cannot measure correctly, since the relation between the sensor path velocities does not follow the spinner anemometer mathematical model (Eqs. 1 to 3).

The $F_{\alpha}$ value calculated for a yawing span of $\pm 60^{\circ}$ was 1.619. This value was used to calibrate the measurements, which are show in Fig. 4a-c. In Fig. 4c, the red line shows the mean wind speed for the measurements where the yawing span is in the range $\pm 60^{\circ}$. Figure $4 \mathrm{~d}$ shows how the 

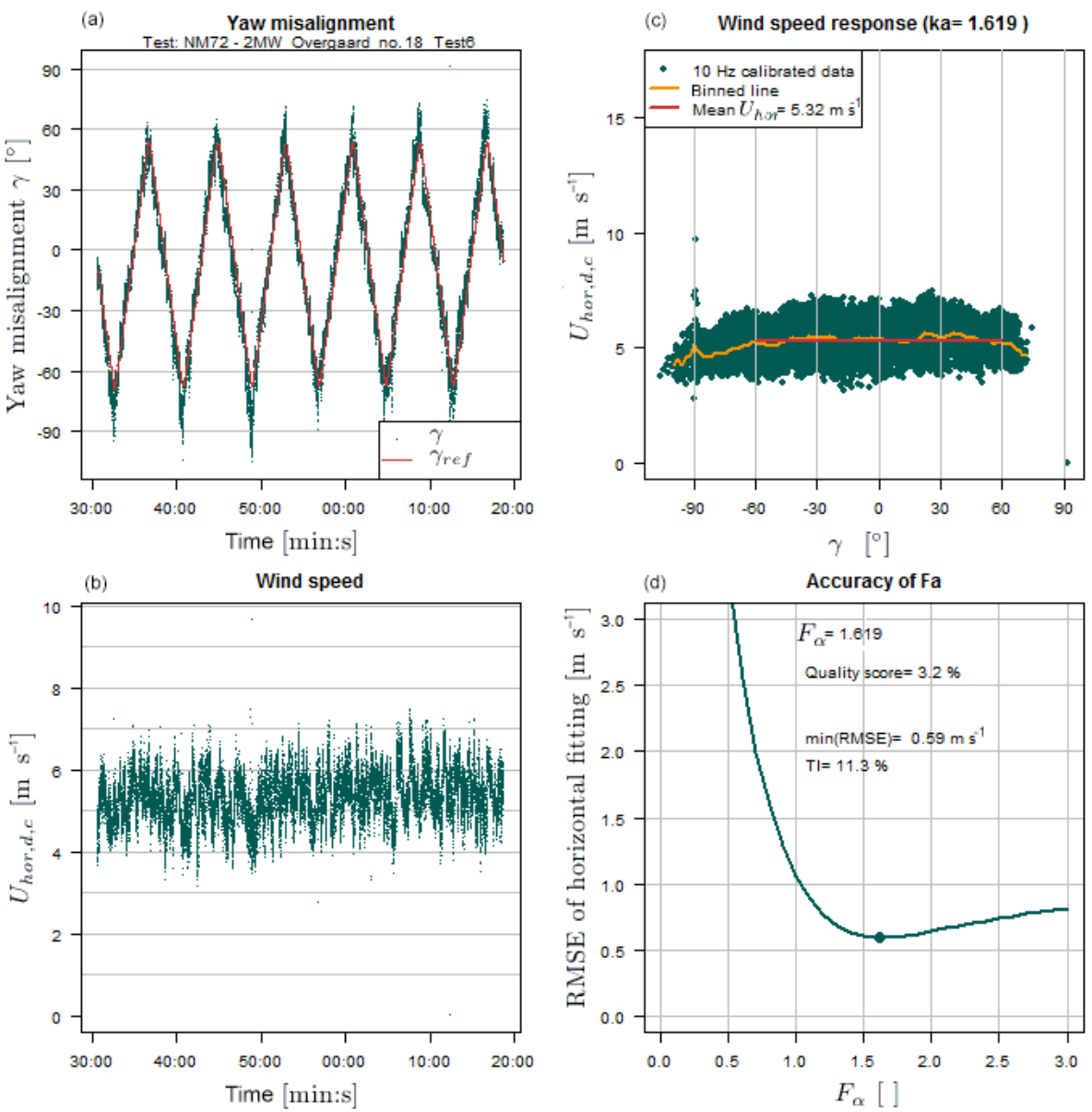

Figure 4. After calibration, test 6. (a) Time series of yaw misalignment as measured by the spinner anemometer and by the yaw position sensor. (b) Wind speed time series as measured by the spinner anemometer before $F_{1}$ calibration and after $F_{\alpha}$ calibration. (c) Wind speed as a function of yaw misalignment both measured by spinner anemometer and calibrated with $F_{\alpha}$. (d) Root mean square error of the horizontal fit (red line in c) as a function of $F_{\alpha}$.

RMSE varies as a function of $F_{\alpha}$, and it also shows the optimum $F_{\alpha}$ as a dot at the minimum RMSE.

The method is based on the assumption of a constant wind speed. When applying the method to a spinner anemometer exposed to natural wind the wind speed will naturally vary in the time frame of about $1 \mathrm{~h}$ needed to complete the six yawing cycles (Fig. 3a). The wind speed variations are clearly visible in the wide scatter of Fig. $3 c$, which are averaged when calculating the RMSE (Eq. 19). The turbulence reduces the repeatability of the result $\left(F_{\alpha}\right)$ since it introduces some randomness into the measurements. The result can be improved by a large number of tests or by using a stable wind source. The worst case is that the increase (and decrease) in wind speed is synchronized with the yaw position of the turbine, which is basically impossible when the turbine is yawed several times.

\section{Sensitivity analysis}

The calibration test was performed several times on the exact same turbine. The rotor was stopped with one blade pointing downwards (the so-called bunny position), and the nacelle was yawed six times for each test: by $\pm 90^{\circ}$ (test 7 to 10 ) or $\pm 60^{\circ}$ (test 1 to 6 ) by operating it manually from the turbine control panel. The yaw moves with a speed of about $0.5^{\circ} \mathrm{s}^{-1}$; therefore, one test of six sweeps takes approximately $1 \mathrm{~h}$. Tests 7 to 10 were made on the same day, one after the other, for the exact same rotor position. The WSR method was used to calculate $F_{\alpha}$ for each test and several yawing spans (Fig. 6); this is also reported in Table 1 for the case of $\pm 60^{\circ}$ yawing span. Test 3 and 5 faced some data acquisition problems and were discarded.

Regarding the ability of the method to give reproducible results, the variation of $F_{\alpha}$ for tests 7 to 10 is within $\pm 2.7 \%$ of the mean value 1.52. Since the rotor position is the same for the four tests, the only possible factor responsible for the 
Table 1. $F_{\alpha}$ values for eight calibration tests made on the same wind turbine. Tests 7 to 10 were made with exact same rotor position relative to a wind turbine yawing span of $\pm 60^{\circ}$.

\begin{tabular}{lcccccccc}
\hline Test & 1 & 2 & 4 & 6 & 7 & 8 & 9 & 10 \\
$F_{\alpha}$ value & 1.63 & 1.72 & 1.73 & 1.62 & 1.46 & 1.53 & 1.48 & 1.53 \\
\hline
\end{tabular}

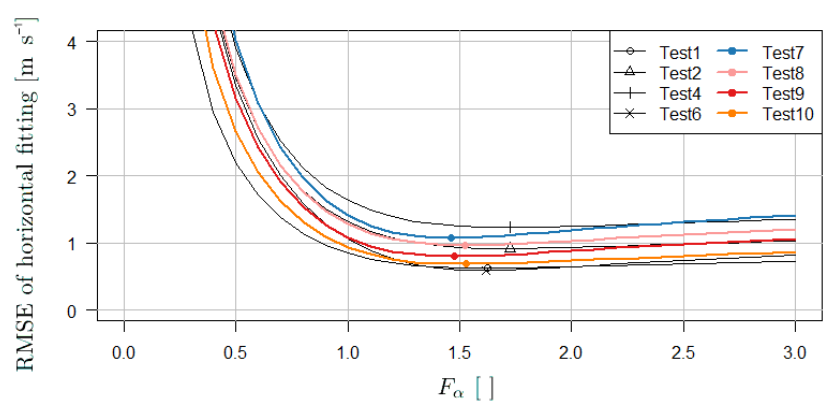

Figure 5. Root mean square error as a function of $F_{\alpha}$. Markers locate the minimum value of RMSE and the corresponding $F_{\alpha}$ value. Bold coloured lines are tests performed for the exact same rotor position.

variations is the wind turbulence. The eight results are within $\pm 8.5 \%$ of the mean value 1.59 . It seems that the $F_{\alpha}$ value relative to the first four tests (about 1.67) is higher than the last four tests (1.50), which could be due to a different rotor position, which plays a role if the rotational symmetry of the spinner and sensor mounting positions is not accurate. The accuracy of the mounting position of the sonic sensors on this spinner was not investigated.

\section{Goodness of a calibration and benchmark on 17 wind turbine models}

The variations encountered in the estimation of $F_{\alpha}$ call for the definition of a variable to judge the quality of the calibration. One indicator could be related to the shape of the curves of Fig. 5. The flatter and shallower the minimum, the larger the uncertainty on $F_{\alpha}$. The indicator was called the quality score (QSC, see Eq. 20), calculated as the slope to the left of the minimum point.

$\mathrm{QSC}=\frac{\operatorname{RMSE}\left(F_{\alpha}-0.1\right)-\operatorname{RMSE}\left(F_{\alpha}\right)}{0.1}$

Figure 7 shows QSC as a function of the span of yawing.

What minimum quality score should a test have to give meaningful $F_{\alpha}$ ? To answer this question, the wind speed response method was applied to a database of yawing tests consisting of 29 calibration tests made on 17 turbine models. Results are shown in Figs. 8 and 9.

Figure 8 can help to identify which conditions of wind speed and turbulence lead to a more precise estimate of $F_{\alpha}$, which means a more steep $\operatorname{RMSE}\left(F_{\alpha}\right)$ curve or, in other

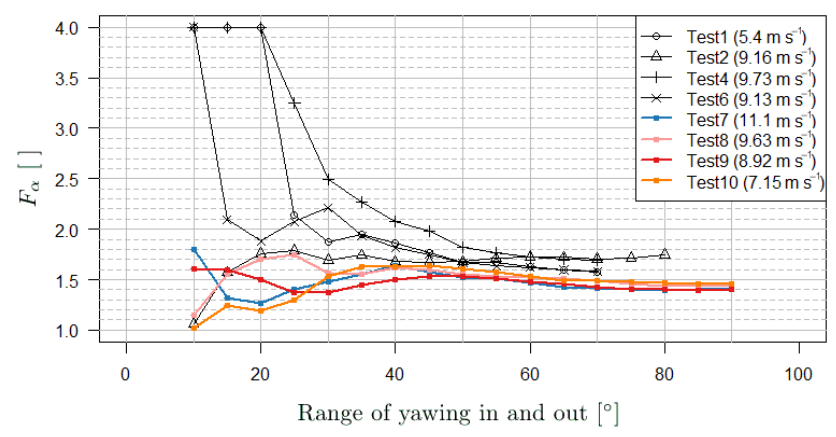

Figure 6. Sensitivity of the $F_{\alpha}$ to the yawing span. Bold coloured lines are tests performed for the exact same rotor position. For test 2 the wind turbine was yawed by $\pm 60^{\circ}$, but an initial offset of the turbine with respect to the wind direction and a wind direction change during the test determined measurements up to $80^{\circ}$. The values in the legend show the mean wind speed during the test.

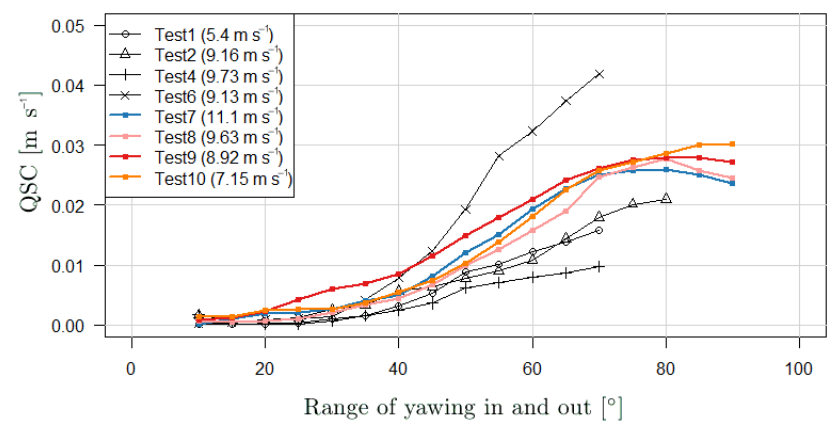

Figure 7. The quality score (QSC) is a measure of how much the RMSE as a function of $F_{\alpha}$ peaks at the minimum. A wide yawing span gives a clearer peak. The values in the legend show the mean wind speed during the test.

words, a high QSC. Average wind speed and turbulence intensity were calculated from the measurements calibrated with $F_{\alpha}$ for a range of yaw misalignments included in the interval -30 to $30^{\circ}$. This is to ensure that there is no flow separation from the spinner surface and therefore ensure the spinner anemometer model validity (the spinner anemometer model is expressed by Eqs. (1)-(3)). Figure 8 shows an inverse relation between the quality score and the turbulence intensity of the wind speed as measured by the spinner anemometer during the yawing test. Figure 8 shows that the QSC increases with the wind speed $U_{\text {hor }}$.

The most pronounced correlation in Fig. 8 is between QSC and TI, where the QSC increases for decreasing turbulence intensity. This suggests that the ideal condition to perform the test is at low turbulence. The initial statement (in Sect. 2) that the wind speed turbulence would reduce the accuracy of the method is also confirmed by a QSC that reduces as the TI increases. A condition of low-turbulent wind can be found by night, when the atmosphere is stable, at a site that is flat with low roughness. It seems also that the QSC increases for 


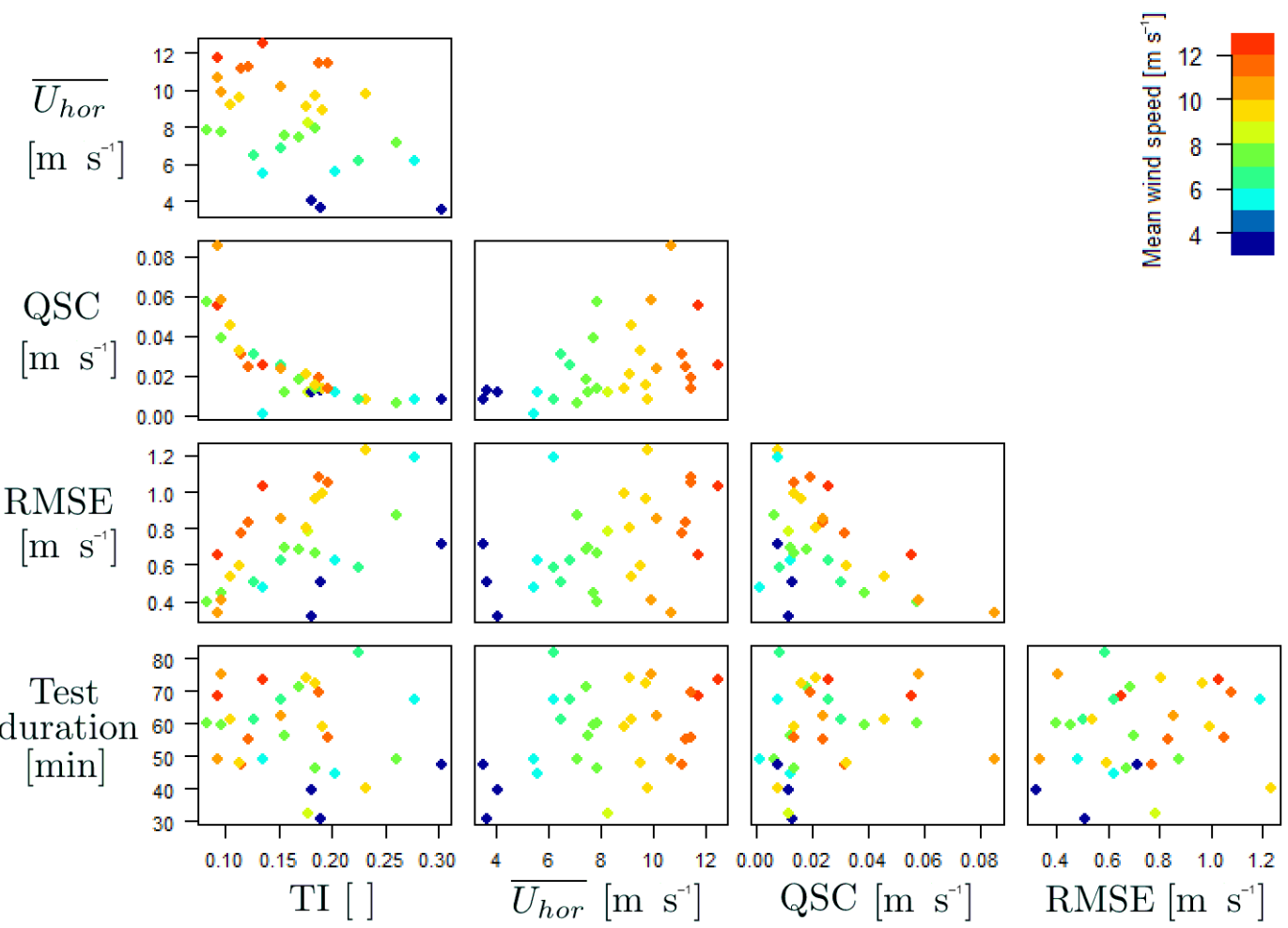

Figure 8. Application of the method to a large database of wind turbines. Colour-coded according to the mean wind speed.
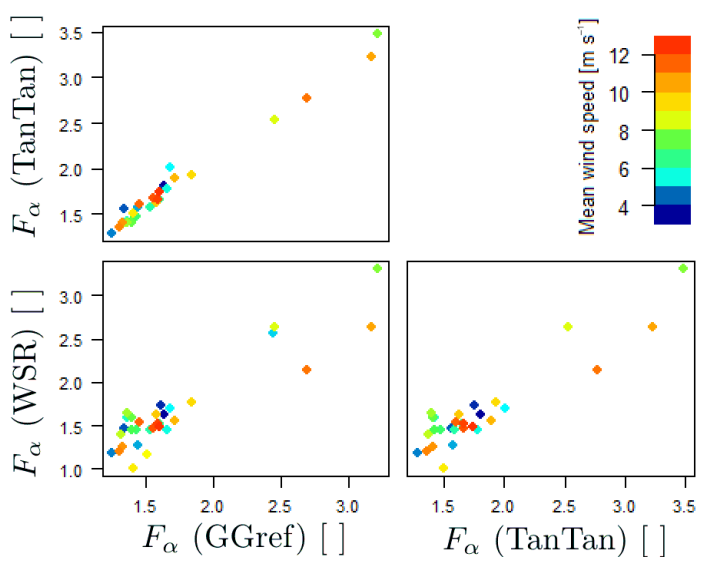

Figure 9. $F_{\alpha}$ calculated with three methods over a large database of wind turbines. Colour-coded according to the mean wind speed.

increasing $U_{\text {hor }}$; however, the scatter of QSC also increases and there are several points with a low QSC despite the high wind speed. This means that to achieve a high QSC, a low TI is more important than a high wind speed.

\section{Comparison with previous methods}

The $F_{\alpha}$ was calculated with the three methods GGref, TanTan, and WSR for a range of yawing $\left(\gamma_{\text {ref }}\right)$, i.e. $\pm 45, \pm 45$ and $\pm 60^{\circ}$, respectively. Figure 9 shows a comparison of $F_{\alpha}$ values for 29 tests made on 17 wind turbine models. All the spinner anemometer were initially set with the same default calibration values $\left(k_{1, \mathrm{~d}}=1, k_{2, \mathrm{~d}}=1\right)$; therefore, it is possible to compare the $F_{\alpha}$ values directly. Most of the turbines present an $F_{\alpha}$ between 1 and 2, values which are attributable to a pointed spinner shape (like a Vestas V52) or a rounded spinner (like a NEG Micon NM80). The four tests with an $F_{\alpha}$ between 2.5 and 3.5 belong to a flat spinner like the one of a Siemens SWT-6.0-154.

The two methods which agrees the most are the GGref and the TanTan methods. This good agreement, however, does not imply that the $F_{\alpha}$ estimate is accurate but rather that the two methods are similar (in fact, they are both based on a linear fitting of the measurements, as described in the section "Existing calibration methods for flow angle measurements").

The value of $F_{\alpha}$ calculated with the WSR method shows a lower level of agreement with the other two methods, being based on a completely different principle.

\section{Conclusions}

The article presented a new method to calibrate spinner anemometer flow angle measurements (yaw misalignment). The advantage of the method is that it does not need the yaw position of the nacelle to be measured. 
The robustness of the method was investigated by repeating the calibration test on the same turbine several times, with the rotor locked in the exact same rotor position to avoid sensor mounting deviations playing a role. The $F_{\alpha}$ values found for four tests for the exact same rotor position were within $\pm 2.7 \%$ of the mean value.

The quality score parameter (QSC) was introduced to quantify the goodness of the $F_{\alpha}$ estimate. The QSC was found inversely dependent on the turbulence intensity. To have a precise estimate of $F_{\alpha}$, it is therefore better to perform the test in low-turbulence wind conditions. The relation found between the QSC and the width of yawing suggests yawing the turbine further than by \pm 60 and up to $\pm 80^{\circ}$ (these values might be different for other spinner shapes). Another issue to consider is that the test could start with an offset and end up being -90 to $70^{\circ}$ instead of -80 to $80^{\circ}$. This is easily avoidable by yawing the wind turbine a bit further than the desired yawing span.

The sensitivity of the method to the width of yawing the turbine in and out of the wind was investigated by applying the calibration method to a subset of the original database. The subset was obtained filtering for $\gamma_{\text {ref }} \in[-s, s]$, where $s$ was the span ranging from 10 to $90^{\circ}$ in steps of $5^{\circ}$. Significant variations of the $F_{\alpha}$ value were found for yawing span $s$ below approximately $60^{\circ}$.
The $F_{\alpha}$ calculated with the wind speed response method was compared with the $F_{\alpha}$ calculated with previous methods (GGref, TanTan) using 29 calibration tests performed by Romo Wind $\mathrm{A} / \mathrm{S}$ on 17 wind turbine models. The sensitivity to the span of yawing showed that the WSR method tends to stabilize to the same values as GGref for a yawing span larger than approximately $50^{\circ}$. Both the GGref and TanTan methods gave similar values of up to $\pm 40^{\circ}$; then, the TanTan method gave a higher $F_{\alpha}$ and diverged from GGref for a yawing span larger than $70^{\circ}$.

A recommended yawing span to use to calculate $F_{\alpha}$ seems to be $\pm 60^{\circ}$ for the WSR method and $\pm 40^{\circ}$ for the TanTan and GGref methods; however, the turbine should be yawed further than this angle $\left( \pm 90^{\circ}\right.$ recommended) to compensate for initial offset error in the yaw position and wind speed direction change during the test.

It is best to perform the test at the lowest possible turbulence intensity, which might be found in stable atmospheric conditions (typically by night) at a flat site with low roughness.

It is recommended to verify the variation of $F_{\alpha}$ as a function of the span of yawing (using the calibrated yaw misalignment if the yaw sensor is not available), since substantially different spinner shapes might give a stable $F_{\alpha}$ at different yawing spans. 


\section{Appendix A: Nomenclature}

$V_{1} \quad$ Speed along the sensor path of probe 1

$V_{2} \quad$ Speed along the sensor path of probe 2

$V_{3} \quad$ Speed along the sensor path of probe 3

$V_{\text {ave }} \quad$ Mean value of $V_{1}, V_{2}, V_{3}$

$U \quad$ Wind speed vector modulus

$U_{\text {hor }} \quad$ Horizontal wind speed component

$U_{\text {hor,d }} \quad$ Horizontal wind speed (non-calibrated)

$U_{\text {hor,d,c }} \quad$ Horizontal wind speed component (calibrated with correct $k_{\alpha}$ but not yet with $k_{1}$ )

$k_{1} \quad$ Calibration constant mainly related to wind speed calibration

$k_{\alpha} \quad$ Calibration constant mainly related to angle calibration

$k_{2} \quad$ Calibration constant (equal to $k_{\alpha} \cdot k_{1}$ )

RMSE Root mean square error

QSC Quality score

GGref Gamma-gamma reference method

$\alpha \quad$ Inflow angle with respect to the shaft axis

$\delta \quad$ Shaft tilt angle

$\beta \quad$ Flow inclination angle

$\gamma \quad$ Yaw misalignment

$\gamma_{\text {ref }} \quad$ Reference yaw misalignment

$\phi \quad$ Rotor position

$\overline{U_{\text {hor }}} \quad$ Mean horizontal wind speed

$\theta \quad$ Azimuth position of flow stagnation point on spinner (relative to sonic sensor 1)

$F_{1} \quad$ Calibration correction factor mainly related to wind speed calibration

$F_{\alpha} \quad$ Calibration correction factor mainly related to angle calibration

$F_{2} \quad$ Calibration correction factor $\left(F_{\alpha} \cdot F_{1}\right)$

TI Turbulence intensity

WSR Wind speed response method

TanTan Tangent-tangent method 
Acknowledgements. We thank Romo wind A/S for financing one third of the $\mathrm{PhD}$ project that this article is part of.

Edited by: H. Hangan

Reviewed by: three anonymous referees

\section{References}

Brent, R. P.: Algorithms for Minimization without Derivatives, Prentice-Hall, DTU Wind Energy, Roskilde, Denmark, 1973.

Demurtas, G., Pedersen, T. F., and Zahle, F.: Calibration of a spinner anemometer for wind speed measurements, Wind Energy, doi:10.1002/we.1965, in press, 2016.
Pedersen, T. F., Madsen, H., Møller, R., Courtney, M., Sørensen, N., Enevoldsen, P., and Egedal, P.: Spinner Anemometry - An Innovative Wind Measurement Concept, European Wind Energy Association (EWEA), 7-10 May 2007, Milan, Italy, 2007.

Pedersen, T. F. and Demurtas, G.: Calibration of a spinner anemometer for flow angle measurements, Wind Energy, I, 305, 2014. 